\title{
Thermochemical Investigations of Nearly Ideal Binary Solvents. 3. Solubility in Systems of Nonspecific Interactions
}

\author{
WIIliam E. Acree, Jr., and Gary L. Bertrand* \\ Department of Chemistry, University of Missouri-Rolla, Rolla, Missouri 65401 (Received September 20, 1976; Revised Manuscript \\ Received April 4, 1977) \\ Publication costs assisted by the University of Missouri-Rolla
}

The simple model which has previously led to successful predictive equations for the partial molar excess enthalpy of a solute in nearly ideal binary solvents has been slightly modified for application to the partial molar excess Gibbs free energy (excess chemical potential) of the solute in these systems. Three predictive equations are derived and tested for their ability to predict solubility in mixed solvents from measurements in the pure solvents. The most successful equation involves volumetrically weighted interaction parameters for the excess Gibbs free energy relative to the Flory-Huggins entropy of mixing, and predicts solubility in 22 systems containing naphthalene, iodine, and stannic iodide as solutes with an average deviation of $1.5 \%$ and a maximum deviation of $4 \%$, using no adjustable parameters.

\section{Introduction}

Recent developments in the investigation of weak association complexes in solution ${ }^{1,2}$ have shown a need for improved approximations for the thermochemical properties of a solute or solutes in a binary solvent system, to allow compensation for the effects of solution nonideality, or, from a slightly different viewpoint, to separate "chemical" and "physical" effects on the properties of the complexes. In order to provide a firm thermodynamic basis for these approximations, much simpler systems must be studied, establishing the qualitative and if possible the quantitative trends of behavior of solutes in binary solvent systems of nonspecific (or physical) interactions.

This work is a continuation of our search for mixing models and equations which will provide reasonable predictions for the thermochemical properties of a solute at high dilution in a binary solvent. Earlier studies ${ }^{3,4}$ have been primarily concerned with the partial molar excess enthalpy of the solute. In this work, we extend our previous consideration of the chemical potential or partial molar Gibbs free energy of the solute ${ }^{4}$ through studies of solubility in binary solvents. Three specific forms of the general predictive equation are compared. It should be noted that these equations are identical in concept and only slightly different in application from equations developed and tested by Heric and co-workers., 5 Similar equations have been developed by O'Connell and Prausnitz ${ }^{7,8}$ and by Nitta and Katayama. ${ }^{9}$ However, we feel that the greater flexibility of our model, and the need to establish the coherence of our model for free energy with our earlier model for the enthalpy of the solute merit this reconsideration of the fundamental equations. Because of the intended application of these approximations to the case of a solute in a complexing solvent, and to the properties of the complex (a second solute), we are particularly concerned with the effects of the relative sizes of the solvent and solute molecules.

We show the development and application of a zeroparameter equation which predicts the solubility of the fairly soluble solute naphthalene in mixtures of small solvents (benzene, carbon tetrachloride, etc.) and those containing a much larger solvent (hexadecane), and of the much smaller and less soluble solute iodine over a similar range of solvent sizes, with a maximum error of $4 \%$ in the 22 systems considered. The application to iodine as solute in these systems of nonspecific interactions is particularly important because of the ability of iodine to form a variety of complexes.

Solubility data for naphthalene in a number of solvent mixtures covering a broad range of molar volumes are available in the literature. The solubility of iodine in several noncomplexing solvent mixtures is also available, but since none of the solvent mixtures contain very large molecules, we have measured the solubility of iodine in two mixtures of $n$-hexadecane with smaller molecules. To provide data for a larger solute molecule, we have also measured the solubility of stannic iodide in cyclohexane + carbon tetrachloride.

\section{Experimental Section}

Fisher Spectroanalyzed carbon tetrachloride, cyclohexane, $n$-heptane, and isooctane were stored over molecular sieves and distilled shortly before use. Eastman Practical Grade $n$-hexadecane was passed through a silica gel column and distilled. Solvent mixtures were prepared by weight, so that mole fractions could be calculated to 0.0001 .

Iodine was Matheson Coleman and Bell Certified ACS Reagent Grade. Stannic iodide was prepared by refluxing metallic tin powder with iodine in chloroform as suggested by Wheatland. ${ }^{10}$ The product was recrystallized three times from hot chloroform, giving a melting point of 144 $\pm 1{ }^{\circ} \mathrm{C}$ (lit. $\mathrm{mp} 144.5^{\circ} \mathrm{C}$ ).

Saturated solutions of stannic iodide in brown glass bottles, protected from light with aluminum foil, were allowed to equilibrate in a constant temperature bath at $25.00 \pm 0.01^{\circ} \mathrm{C}$ for several days. The attainment of equilibrium was verified by repetitive measurements after several additional days. The solubility was measured by transferring an aliquot of solution through a coarse filter into a tared container for analysis by the gravimetric procedure used by Dorfman and Hildebrand. ${ }^{11}$ Iodine solutions were equilibrated in a similar fashion, transferring a weighed aliquot to a flask containing a known excess of aqueous arsenic trioxide, then back-titrated with a freshly standardized iodine solution to the starch endpoint. Solubilities were reproducible to within $1 \%$. Results are reported in Table I. In the pure solvents our results were in good agreement with previously reported values, as presented in Table II. 
TABLE I: Observed and Calculated Values of the Solubility of Iodine and Stannic Iodide in Some Binary Solvents at $25{ }^{\circ} \mathrm{C}$

\begin{tabular}{|c|c|c|c|c|c|}
\hline \multirow[b]{2}{*}{ Solute/solvent $(1)+$ solvent $(2)$} & \multirow[b]{2}{*}{$X_{1}^{0}$} & \multirow[b]{2}{*}{$X_{\text {solute }}^{\text {sat }}$} & \multicolumn{3}{|c|}{$\%$ deviations of calcd values ${ }^{a}$} \\
\hline & & & $(\mathrm{XX})$ & $(\mathrm{XV})$ & $(\mathrm{VV})$ \\
\hline \multirow{5}{*}{$\mathrm{I}_{2} / n-\mathrm{C}_{16} \mathrm{H}_{34}+n-\mathrm{C}_{7} \mathrm{H}_{16}$} & 0.0815 & 0.00762 & -4.6 & +1.0 & -0.6 \\
\hline & 0.1429 & 0.00809 & -6.4 & +2.1 & -0.5 \\
\hline & 0.1668 & 0.00816 & -5.8 & +3.8 & +1.0 \\
\hline & 0.3267 & 0.00958 & -11.2 & +2.5 & -1.1 \\
\hline & 0.6164 & 0.01174 & -10.7 & +1.9 & -1.5 \\
\hline \multirow[t]{5}{*}{$\mathrm{I}_{2} / n-\mathrm{C}_{16} \mathrm{H}_{34}+i-\mathrm{C}_{8} \mathrm{H}_{18}$} & 0.1015 & 0.00681 & -4.3 & +1.5 & -0.6 \\
\hline & 0.1563 & 0.00735 & -7.6 & +0.4 & -1.7 \\
\hline & 0.2169 & 0.00778 & -7.9 & +2.0 & -0.5 \\
\hline & 0.3554 & 0.00896 & -9.9 & +2.3 & -0.7 \\
\hline & 0.5800 & 0.01086 & -9.3 & +2.1 & -0.6 \\
\hline \multirow{4}{*}{$\mathrm{SnI}_{4} / \mathrm{CCl}_{4}+\mathrm{c}-\mathrm{C}_{6} \mathrm{H}_{12}$} & 0.1491 & 0.00823 & -1.3 & -1.8 & -1.7 \\
\hline & 0.3617 & 0.00962 & -0.6 & -1.3 & -1.2 \\
\hline & 0.6183 & 0.01155 & -0.6 & -1.2 & -1.1 \\
\hline & 0.8676 & 0.01357 & -0.5 & -0.7 & -0.6 \\
\hline
\end{tabular}

a \% deviation $=100 \ln \left(X_{\text {calcd }}^{\text {sat }} / X_{\text {obsd }}^{\text {sat }}\right)$.

TABLE II: Comparison with Literature Values of the Solubilities of Iodine and Stannic Iodide in Pure Solvents at $25^{\circ} \mathrm{C}$

\begin{tabular}{llcc}
\hline & & \multicolumn{2}{c}{$\begin{array}{c}\text { (Mole fraction } \\
\text { solubility) } \times 10^{3}\end{array}$} \\
\cline { 3 - 5 } Solute & \multicolumn{1}{c}{ Solvent } & Exptl & Lit. \\
\hline Iodine & Isooctane & 5.923 & $5.92^{a}$ \\
Iodine & $n$-Heptane & 6.912 & $6.916^{b}, 6.79^{c}$ \\
Iodine & $n$-Hexadecane & 14.28 & $14.50^{d}$ \\
Stannic iodide & CCl $_{4}$ & 14.63 & $14.599^{e} 14.4^{f}$ \\
Stannic iodide & Cyclohexane $^{f}$ & 7.216 & $7.273^{g}$
\end{tabular}

$a^{a}$ G. R. Negishi, L. H. Donnally, and J. H. Hildebrand, J. Am. Chem. Soc., 55, $4793(1933)$. ${ }^{b}$ G. Kortüm and V. M. Vogel, Z. Elektrochem., 59, 16 (1955). ${ }^{c}$ J. H. Hildebrand and C. A. Jenks, J. Am. Chem. Soc., 42, 2180 (1920). ${ }^{d}$ K. Shinoda and J. H. Hildebrand, J. Phys. Chem., 69, 605 (1965). ${ }^{e}$ See ref 11. $f$ B. E. Smith and J. Walkley, Trans. Faraday Soc., 56, 220 (1969). ' See ref 14 .

\section{Discussion}

Burchfield and Bertrand ${ }^{4}$ developed general equations for the partial molar excess properties of a solute at high dilution in a binary solvent for a simple model system obeying a ternary mixing equation

$$
\begin{aligned}
& \Delta Z_{123}^{\mathrm{ex}}=\left(n_{1} \Gamma_{1}+n_{2} \Gamma_{2}+n_{3} \Gamma_{3}\right)^{-1}\left(n_{1} \Gamma_{1} n_{2} \Gamma_{2} A_{12}\right. \\
& \left.\quad+n_{1} \Gamma_{1} n_{3} \Gamma_{3} A_{13}+n_{2} \Gamma_{2} n_{3} \Gamma_{3} A_{23}\right)
\end{aligned}
$$

in which $Z$ represents any extensive thermodynamic property described in terms of interaction parameters $\left(A_{i j}\right)$ and weighting factors $\left(\Gamma_{i}\right)$. The partial molar excess properties of the solute at infinite dilution in mixtures $\left[\left(\Delta \bar{Z}_{3}{ }^{\mathrm{ex}}\right)^{*}\right]$ are shown to depend on a weighted average of the properties in the pure solvents $\left[\left(\Delta \bar{Z}_{3}{ }^{\mathrm{ex}}\right) *{ }_{X_{1}}{ }=1\right.$, $\left.\left(\Delta \bar{Z}_{3}{ }^{\mathrm{ex}}\right)^{*}{ }_{X_{2}{ }^{0}=1}\right]$ and a contribution due to the "unmixing" of the solvent pair by the presence of the solute:

$$
\begin{aligned}
& \left(\Delta \bar{Z}_{3}^{\mathrm{ex}}\right)^{*}=f_{1}^{0}\left(\Delta \bar{Z}_{3}^{\mathrm{ex}}\right)^{*}{ }_{X_{1}{ }^{0}=1}+f_{2}{ }^{0}\left(\Delta \bar{Z}_{3}^{\mathrm{ex}}\right)^{*}{ }_{X_{2}{ }^{0}=1} \\
& -\Gamma_{3}\left(X_{1}^{0} \Gamma_{1}+X_{2}^{0} \Gamma_{2}\right)^{-1}\left(\Delta \bar{Z}_{12}^{\mathrm{ex}}\right) \\
& f_{1}^{0}=\left(1-f_{2}^{0}\right)=n_{1} \Gamma_{1} /\left(n_{1} \Gamma_{1}+n_{2} \Gamma_{2}\right) \\
& X_{1}^{0}=\left(1-X_{2}^{0}\right)=n_{1} /\left(n_{1}+n_{2}\right)
\end{aligned}
$$

Burchfield and Bertrand considered various approximations for weighting factors and concluded that the most accurate predictions for heats of solution could be obtained with weighting factors based on the mixing properties of the binary systems of solvents and solute, but the use of molar volumes for weighting factors provided reasonably accurate predictions. The superiority of the use of molar volumes over the simpler approximation of equating all three weighting factors appeared to derive from the effect of weighting factors on the "unmixing" term of eq 2 rather than from the effect on the weighted average of the properties of the solute in the pure solvents. Enhancement of the unmixing term by a large solute molecule can lead to predictions of maxima or minima of the properties of the solute in simple mixtures. It should be noted that precise applicability of eq 2 to a thermochemical property and its temperature and pressure derivatives $(G$ and $H$ or $S, G$ and $V, H$ and $C_{P}$ ) requires that weighting factors be independent of temperature and pressure. Therefore, molar volumes and other experimentally determined weighting factors must be regarded as approximations to these "true" weighting factors, and for application to conditions of varying temperature and pressure, they should be referred to a specified condition, such as $25^{\circ} \mathrm{C}$ and $1 \mathrm{~atm}$, or to an extrapolated state such as a "closepacked" volume.

Interaction parameters and weighting factors are rather straightforward in the case of directly observed excess properties such as enthalpy and volume. In the case of Gibbs free energy, however, the total free energy of mixing is observed and the excess is calculated as the difference between the observed value and the value for an ideal solution of the same composition

$\Delta G^{\text {mixing }}=R T \sum_{i=1}^{N} n_{i} \ln X_{\mathrm{i}}+\Delta G^{\mathrm{ex}}$

For mixtures of molecules with considerably different molar volumes, excess mixing equations of the form of eq 1 more accurately describe the difference between the observed free energy of mixing and that predicted by the Flory-Huggins equation

$\Delta G^{\mathrm{mixing}}=R T \sum_{i=1}^{N} n_{i} \ln \Phi_{i}+\Delta G^{\mathrm{fh}}$

in which $\phi_{i}$ represents a weighted mole fraction based on weighting factors which are similar to molar volumes, but which do not vary with temperature or pressure; and $\Delta G^{\text {th }}$ has the same form as eq 1 but with specific weighting factors $\Gamma_{i}^{\text {fh }}$ and interaction parameters $A_{i j}$ fh ${ }_{12}$ Treatment of these mixing equations in the manner of Burchfield and Bertrand ${ }^{4}$ leads to two general equations for approximating the partial molar excess Gibbs free energy of a solute at high dilution in a binary solvent:

$$
\begin{aligned}
\Delta \bar{G}_{3}^{\mathrm{ex}} & =\left(1-f_{3}\right)^{2}\left[f_{1}{ }^{0}\left(\Delta \bar{G}_{3}^{\mathrm{ex}}\right)^{*}{ }_{X_{1}^{0}=1}+f_{2}{ }^{o}\left(\Delta \bar{G}_{3}^{\mathrm{ex}}\right)^{*}{ }_{X_{2}{ }^{0}=1}\right. \\
& \left.-\Gamma_{3}\left(X_{1}^{0} \Gamma_{1}+X_{2}^{0} \Gamma_{2}\right)^{-1}\left(\Delta \bar{G}_{12}^{\mathrm{ex}}\right)\right]
\end{aligned}
$$


TABLE III: Comparison of Predictive Equations for the Solubilities of Naphthalene, Iodine, and Stannic Iodide in Various Binary Systems

\begin{tabular}{|c|c|c|c|c|c|c|}
\hline \multirow[b]{2}{*}{ Solute } & \multirow[b]{2}{*}{ Solvent } & \multirow[b]{2}{*}{$\operatorname{Ref}$} & \multicolumn{3}{|c|}{$\begin{array}{l}\text { RMS deviations }{ }^{u}(\%) \text { for the } \\
\text { predictive eq }\end{array}$} & \multirow{2}{*}{$\begin{array}{l}\Delta G_{12}^{\mathrm{ex}} \\
\operatorname{Ref}\end{array}$} \\
\hline & & & $(\mathrm{XX})$ & $(\mathrm{XV})$ & $(V V)$ & \\
\hline Naphthalene & Benzene + cyclohexane & $a$ & -1.4 & -1.2 & -1.1 & $i$ \\
\hline Naphthalene & Benzene $+\mathrm{CCl}_{4}$ & $a$ & +1.5 & +1.7 & +1.9 & $i$ \\
\hline Naphthalene & Benzene + hexane & $a$ & +2.3 & 1.4 & 1.4 & $d$ \\
\hline Naphthalene & Cyclohexane + hexadecane & $b$ & -4.0 & +2.9 & +1.4 & $j$ \\
\hline Naphthalene & Hexane + hexadecane & $b$ & -6.8 & +1.8 & +0.8 & $o$ \\
\hline Naphthalene & $\mathrm{CCl}_{4}+$ cyclohexane & $b$ & 0.3 & -0.5 & -0.6 & $i$ \\
\hline Naphthalene & $\mathrm{CCl}_{4}+$ hexadecane & $b$ & +8.0 & +4.5 & +2.4 & $p$ \\
\hline Naphthalene & Benzene + hexadecane & $a$ & +9.2 & +2.4 & +0.7 & $p$ \\
\hline Naphthalene & $\mathrm{CCl}_{4}+$ hexane & $b$ & +3.2 & 1.3 & 0.5 & $h$ \\
\hline Naphthalene & Cyclohexane + hexane & $b$ & $0 . \overline{8}$ & 0.6 & 0.6 & $e$ \\
\hline Naphthalene & Benzene + toluene & $a$ & +0.5 & +0.5 & +0.6 & $q$ \\
\hline Naphthalene & $\mathrm{CCl}_{4}+$ toluene & $c$ & -1.5 & -1.5 & -1.5 & $k$ \\
\hline Naphthalene & Cyclohexane + toluene & $c$ & -1.8 & -0.9 & -0.8 & $f$ \\
\hline Naphthalene & Ethylbenzene $+\mathrm{CCl}_{4}$ & $c$ & 0.5 & 0.7 & 0.6 & $w$ \\
\hline Naphthalene & Ethylbenzene + cyclohexane & $c$ & -1.7 & 0.3 & 0.3 & $w$ \\
\hline Iodine & Cyclohexane + hexane & $m$ & +1.7 & -1.5 & -2.5 & $e$ \\
\hline Iodine & $\mathrm{CCl}_{4}+$ hexane & $m$ & +5.0 & -1.3 & -2.0 & $h$ \\
\hline Iodine & Hexadecane + heptane & $v$ & -8.2 & +2.4 & -1.0 & $l$ \\
\hline Iodine. & Hexadecane + isooctane & $v$ & -8.1 & +1.8 & -0.9 & $r$ \\
\hline Iodine & Cyclohexane + heptane & $n$ & +2.1 & -0.5 & -0.8 & $f$ \\
\hline $\mathrm{SnI}_{4}$ & Cyclohexane + benzene & $g$ & 2.2 & 2.8 & 3.1 & $i$ \\
\hline $\mathrm{SnI}_{4}$ & Cyclohexane $+\mathrm{CCl}_{4}$ & $v$ & -0.8 & -1.3 & -1.2 & $i$ \\
\hline
\end{tabular}

Properties used in calculations

\begin{tabular}{|c|c|c|c|c|c|}
\hline Solvent & $\bar{V}^{0}, \mathrm{~mL} / \mathrm{mol}$ & Solvent & $\bar{V}^{\circ}, \mathrm{mL} / \mathrm{mol}$ & Solute & $a_{3}^{\text {solid }}$ \\
\hline Benzene & 89.41 & Iodine & 59.593 & Iodine & $0.258^{s}$ \\
\hline $\mathrm{CCl}_{4}$ & 97.08 & Isooctane & 166.09 & Naphthalene & $0.312^{a}$ \\
\hline Cyclohexane & 108.76 & Naphthalene & 123.00 & Stannic iodide & $0.1127^{t}$ \\
\hline Hexadecane & 294.12 & Stannic iodide & 151.00 & & \\
\hline Heptane & 147.48 & Toluene & 106.84 & & \\
\hline Hexane & 131.59 & Ethylbenzene & 123.06 & & \\
\hline
\end{tabular}

${ }^{a}$ See ref 5a. ${ }^{b}$ See ref 5c. ${ }^{c}$ See ref 5b. ${ }^{d}$ V. C. Smith and R. L. Robinson, Jr., J. Chem. Eng. Data, 15, $391(1970)$

I. P.-C. Li, B. C.-Y. Lu, and E. C. Chen, ibid., 18, $305(1973)$. f T. Katayama, E. K. Sung, and E. N. Lightfoot, $A I C h E J$, 11,294 (1965). ${ }^{g}$ See ref $14 .{ }^{h}$ D. V. S. Jain, V. K. Gupta, and B. S. Lark, Can. J. Chem., 8, 815 (1970). i J. R. Goates, R. J. Sullivan, and J. B. Ott, J. Phys. Chem., 63, 589 (1959). j J. D. Gomez-Ibanez and J. J. C. Shieh, ibid., 69, 1660 (1965). ${ }^{k}$ R. P. Rastogi, J. Nath, and J. Misra, ibid., 71, 1277 (1967). ${ }^{l}$ J. N. Bronsted and J. K. Koefoed, Dank. Videnskab. Selskab. (Mat. Fys. Medd.), 22, 1 (1946). $m$ See ref 15b. ${ }^{n}$ G. Kortüm and V. M. Vogel, Z. Elektrochem., 59, 16 (1955). ${ }^{\circ}$ M. L. McGlashan and A. G. Williamson, Trans. Faraday Soc., 57, 588 (1961). p D. V. S. Jain and B. S. Lark, J. Chem. Thermodyn., 5, 455 (1973). q J. S. Rowlinson, "Liquids and Liquid Mixtures", Academic Press, New York, N.Y., 1959, p 150. r Footnote $q, \mathrm{p} \mathrm{159.} \mathrm{s} \mathrm{See} \mathrm{ref} \mathrm{8,} \mathrm{p} \mathrm{142.} \mathrm{'t} \mathrm{S.} \mathrm{K.} \mathrm{Suri} \mathrm{and} \mathrm{V.} \mathrm{Ramakrishna,} \mathrm{Can.} \mathrm{J.} \mathrm{Chem.,} \mathrm{47,} \mathrm{3049} \mathrm{(1969).}$ ${ }^{u}$ RMS deviation $(\%)=\left(100 / N^{1 / 2}\right)\left\{\Sigma\left[\ln \left(X_{c a l c d}^{\text {sat }} / X_{\text {expt }}^{\text {sat }}\right)\right]^{2}\right\}^{1 / 2}$; an algebraic sign indicates that all deviations were of the same sign. ${ }^{v}$ This work. ${ }^{w}$ D. V. S. Jain and O. P. Yadav, Indian J. Chem., 12, 718 (1974).

and

$$
\begin{aligned}
& \Delta \bar{G}_{3}^{\mathrm{fh}}=\left(1-f_{3}\right)^{2}\left[f_{1}^{0}\left(\Delta \bar{G}_{3}^{\mathrm{fh}}\right)^{*}{ }_{X_{1}^{0}=1}+f_{2}^{0}\left(\Delta \bar{G}_{3}^{\mathrm{fh}}\right)^{*}{ }_{X_{2}{ }^{0}=1}^{\mathrm{fh}}\left(X_{1}^{0} \Gamma_{1}^{\mathrm{fh}}+X_{2}^{0} \Gamma_{2}^{\mathrm{fh}}\right)^{-1}\left(\Delta \bar{G}_{12}^{\mathrm{fh}}\right)\right] \\
& -\Gamma_{3}^{\mathrm{f}}(7)
\end{aligned}
$$

In eq 2,6 , and 7 , and the following equations, the asterisk indicates an extrapolated value for the infinitely dilute solution $\left(f_{3}^{*}=0\right)$. We have considered evaluation of weighting factors $\left(\Gamma_{i}\right.$ and $\Gamma_{i}^{\text {fh }}$ ) from experimental data, as was done for predicting heats of solution in binary solvents. However, it is clear that weighting factors derived from free energy data are of less utility in predictive equations than are the simple approximations for weighting factors. In addition, calculated weighting factors for the excess free energy of mixing of binary systems are often found to be strongly dependent on temperature. This condition probably arises from the fact that the interaction parameters for the free-energy functions are usually small differences between much larger interaction parameters for enthalpy and entropy. Small changes in the interaction parameters and weighting factors for entropy and enthalpy can show up as large relative changes in the corresponding parameters for the free energy. Thus, to provide a proper "count" of the relative numbers of interactions of the solute with the individual solvents, we feel that weighting factors based on the excess enthalpy or entropy would be more appropriate than those based on the free energy of mixing. However, we do not feel that the relatively small amount of data presently available can provide a conclusive test of these ideas. Also, our main concern at the present time is to find the simplest equations which require the least amount of experimental data to adequately predict the binary solvent effect on the Gibbs free energy of a solute in simple systems. Since we have found approximations which perform adequately for the data available, the introduction of more complex approximations does not seem to be warranted.

The general equation (eq 6 and 7) for the excess partial molar properties of the solute in the binary solvent can be related to the solubility through the basic thermodynamic relationships

$$
\begin{aligned}
& \left(\Delta \bar{G}_{3}^{\text {ex }}\right)=R T \ln \left(a_{3}{ }^{\text {solid }} / X_{3}{ }^{\text {sat }}\right) \\
& \left(\Delta \bar{G}_{3}^{\text {fh }}\right)=R T\left\{\ln \left(a_{3}{ }^{\text {solid }} / \Phi_{3}{ }^{\text {sat }}\right)-\left[1-\left(\bar{V}_{3}^{0} / \bar{V}\right)\right]\right\}
\end{aligned}
$$

in which $a_{3}{ }^{\text {solid }}$ is the activity of the solid solute. It is defined as the ratio of the fugacity of the solid to the fugacity of the pure supercooled liquid. ${ }^{8}$ Application of eq 6 and 8 or eq 7 and 9 to the solubilities of the solute in the pure solvents gives values of $\left(\Delta \bar{G}_{3}{ }^{\mathrm{ex}}\right)^{*} X_{1}{ }^{0}=1$ and 
$\left(\Delta \bar{G}_{3}{ }^{\mathrm{ex}}\right) *{ }_{X_{2}=1}{ }^{0}$, or $\left(\Delta \bar{G}_{3}^{\text {fh }}\right){ }_{X_{1}{ }^{0}=1}$ and $\left(\Delta \bar{G}_{3}^{\text {fh }}\right){ }^{*}{ }_{X_{2}=1}$, if the appropriate weighting factors are known. We have considered three equations based on different types of weighting factors: (XX) based on eq 6 with all weighting factors equal, (XV) based on eq 6 with molar volumes as weighting factors, and (VV) based on eq 7 with molar volumes as weighting factors. A fourth permutation of these equations (VX) has little theoretical justification and was not considered. The three predictive equations are

$$
\begin{aligned}
& R T \ln \left(a_{3}{ }^{\text {solid }} / X_{3}{ }^{\text {sat }}\right)=\left(1-X_{3}{ }^{\text {sat }}\right)^{2}\left[X_{1}{ }^{0}\left(\Delta \bar{G}_{3}^{\text {ex }}\right)^{*} X_{1}{ }^{0}=1\right. \\
& \left.+X_{2}^{0}\left(\Delta \bar{G}_{3}^{\text {ex }}\right)_{X_{2}^{0}=1}-\left(\Delta \bar{G}_{12}^{\text {ex }}\right)\right] \\
& R T \ln \left(a_{3}{ }^{\text {solid }} / X_{3}^{\text {sat }}\right)=\left(1-\Phi_{3}^{\text {sat }}\right)^{2}\left[\Phi_{1}{ }^{0}\left(\Delta \bar{G}_{3}^{\text {ex }}\right)^{*}{ }_{X_{1}{ }^{0}=1}\right. \\
& +\Phi_{2}{ }^{0}\left(\Delta \bar{G}_{3}^{\mathrm{ex}}\right)^{*}{X_{2}{ }^{0}=1}_{1}-\bar{V}_{3}^{0}\left(X_{1}^{0} \bar{V}_{1}^{0}\right. \\
& \left.\left.+X_{2}^{0} \bar{V}_{2}^{0}\right)^{-1}\left(\Delta \bar{G}_{12}^{\mathrm{ex}}\right)\right] \\
& R T\left[\ln \left(a_{3}{ }^{\text {solid }} / \Phi_{3}^{\text {sat }}\right)-\left(1-\frac{\bar{V}_{3}^{0}}{X_{1}^{0} \bar{V}_{1}^{0}+X_{2}^{0} V_{2}^{0}}\right)(1\right. \\
& \left.\left.-\Phi_{3}^{\text {sat }}\right)\right]=\left(1-\Phi_{3}^{\text {sat }}\right)^{2}\left[\Phi_{1}^{0}\left(\Delta \bar{G}_{3}^{\text {fh }}\right)^{*} X_{1}^{0}=1\right. \\
& +\Phi_{2}{ }^{0}\left(\Delta \bar{G}_{3}^{\text {fh }}\right)^{*} X_{2}^{0}=1-\bar{V}_{3}^{0}\left(X_{1}^{0} \bar{V}_{1}^{0}\right. \\
& \left.\left.+X_{2}^{0} \bar{V}_{2}^{0}\right)^{-1}\left(\Delta \bar{G}_{12}^{\mathrm{fh}}\right)\right]
\end{aligned}
$$

With these equations, solubility data measured in pure solvents can be used to calculate the excess partial molar Gibbs free energy (or excess over the Flory-Huggins prediction) of the solute at infinite dilution in these pure solvents, then these values can be combined with the excess free energy of the binary solvent ${ }^{9}$ to predict the solubility in mixtures without the use of any adjustable parameters.

The predictive abilities of these equations are compared in Table III for 22 systems for which solubility data and the excess free energy of the solvent pair were available at or near the same temperature. Equation VV, based on molar volumes as weighting factors for the excess free energy over the Flory-Huggins entropy, is seen to be the most generally applicable with an overall average ( $\mathrm{rms}$ ) deviation of $1.5 \%$, and a maximum error for a single data point of $4 \%$. This maximum deviation occurs in a system ( $\mathrm{SnI}_{4}$-benzene + cyclohexane) in which complex formation has been suggested. ${ }^{13}$ The primary advantage of eq VV over the other two predictive equations is in its applicability to the hexadecane systems. If these systems are excluded, (XV) is slightly better overall than eq VV, but eq $\mathrm{XX}$ is significantly poorer than the other two equations. This behavior is in agreement with the findings of Burchfield and Bertrand, ${ }^{4}$ who concluded that volumebased weighting factors gave better predictions for the partial molar excess enthalpy of the solute than did the simple mole fraction parameters. It should be noted that differentation of eq 6 and 7 with respect to temperature leads to identical forms of the equation for excess enthalpy.

The success of eq VV in predicting the binary solvent effect on solubilities as large as 0.35 volume fraction in solvents with molar volumes covering a threefold range and for solutes with molar volumes ranging from 60 to 150 $\mathrm{mL} / \mathrm{mol}$ indicates that this equation will form an adequate base for approximations of the "physical" interactions in a system containing "chemical" interactions such as those between a solute and a complexing solvent. Three treatments of solubility in complexing systems have been reported. Purkayastha and Walkley ${ }^{14}$ treated the solubility of iodine in binary solvents containing benzene in a "physical" manner, using a modified form of the solubility parameter approach. Sytilin ${ }^{15}$ has treated the solubility of iodine in all solvent mixtures, including those of purely "inert" solvents such as $n$-hexane + cyclohexane, in terms of solvational complexes. The true situation must lie intermediate between these two extremes. Nitta and Katayama"d used a combination of "physical" and "chemical" effects to correlate the solubility of iodine in mixtures of benzene + cyclohexane and benzene + carbon tetrachloride. However, their use of a solvent-independent equilibrium constant and a parameterized form of the excess free energy of the solvent pair necessitated the use of an additional parameter in the form of an equilibrium constant for the benzene-carbon tetrachloride complex. It is hoped that our method of using the measured excess free energy of the solvent pair will avoid the problem of dealing with complexes between the binary solvents, and will allow approximations for dealing with the possible solvent dependence of the equilibrium constant for solute complexes.

\section{References and Notes}

(1) G. L. Bertrand, J. Phys. Chem., 79, 48 (1975)

(2) G. L. Bertrand and T. E. Burchfield, "Analytical Calorimetry", Vol. 3, R. S. Porter and J. F. Johnson, Ed., Plenum Press, New York, N.Y., 1974 , p 283.

(3) E. L. Taylor and G. L. Bertrand, J. Solution Chem., 3, 479 (1974).

(4) T. E. Burchfield and G. L. Bertrand, J. Solution Chem., 4, 205 (1975).

(5) (a) E. L. Heric and C. D. Posey, J Chem. Eng. Data, 9, 35 (1964); (b) ibid, 9, 161 (1964); (c) ibid., 10, 25 (1965).

(6) E. L. Heric and K. Yeh, J. Chem. Eng. Data, 15, 13 (1970).

(7) J. P. O'Connell and J. M. Prausnitz, Ind. Eng. Chem., Fundam., 3, 347 (1964)

(8) J. H. Hildebrand, J. M. Prausnitz, and R. L. Scott, "Regular and Related Solutions", Van Nostrand-Reinhold, New York, N.Y., 1970 p 142.

(9) (a) T. Nitta and T. Katayama, J. Chem. Eng. Jpn., 6, 1 (1973); (b) ibid., 6, 298 (1973); (c) lbid., 7, 310 (1974); (d) ibid., 8, 175 (1975).

(10) D. A. Wheatland, J. Chem. Ed., 50, 854 (1973).

(11) M. E. Dorfman and J. H. Hildebrand, J. Am. Chem. Soc., 49, 729 (1927).

(12) For a binary solvent mixture, the excess molar Gibbs free energy over the predictions of the Flory-Huggins equation is related to the defined excess free energy by

$$
\begin{aligned}
& \Delta \bar{G}_{12}^{\text {th }}=\Delta \bar{G}_{12}^{\mathrm{eX}}+R T \ln \left[\left(X_{1}{ }^{0} \bar{V}_{1}^{0}\right.\right.
\end{aligned}
$$

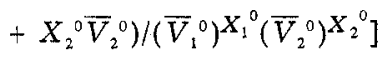

(13) J. F. Murphy and D. E. Baker, Can. J. Chem., 43, 1272 (1965)

(14) A. Purkayastha and J. Walkley, Can.J. Chem., 50, 834 (1972)

(15) (a) M. S. Sytlin, Russ. J. Phys. Chem., 48, 1091 (1974); (b) ibid., 48, 1353 (1974); (c) ibid., 48, 1500 (1974) 